PAPER

The effects of electron cyclotron heating and current drive on toroidal Alfvén eigenmodes in tokamak plasmas

To cite this article: S E Sharapov et al 2018 Plasma Phys. Control. Fusion 60014026

View the article online for updates and enhancements.
Related content

- Energetic particle physics in fusion
$\frac{\underline{\text { research in preparation for burning plasma }}}{\text { experiments }}$
N.N. Gorelenkov, S.D. Pinches and K. Toi
- Energetic particle instabilities in fusion
plasmas
S.E. Sharapov, B. Alper, H.L. Berk et al.
- Energetic particles in spherical tokamak
plasmas
K G McClements and E D Fredrickson

Recent citations

- Kinetic equilibrium reconstruction for the
NBI- and ICRH-heated H-mode plasma on
EAST tokamak
Zhen ZHENG et al 


\title{
The effects of electron cyclotron heating and current drive on toroidal Alfvén eigenmodes in tokamak plasmas
}

\author{
S E Sharapov ${ }^{1}$ (1), M Garcia-Munoz ${ }^{2}$, M A Van Zeeland ${ }^{3}$, B Bobkov ${ }^{4}$, \\ I G J Classen ${ }^{5}$, J Ferreira ${ }^{6}$, A Figueiredo $^{6}$, M Fitzgerald $^{1}$, \\ J Galdon-Quiroga ${ }^{2}$ (0), D Gallart ${ }^{7}$, B Geiger ${ }^{4}$, J Gonzalez-Martin ${ }^{2}$, \\ T Johnson ${ }^{8}$, P Lauber ${ }^{4}$, M Mantsinen ${ }^{7,9}$, F Nabais $^{6}$, V Nikolaeva ${ }^{4}$, \\ M Rodriguez-Ramos ${ }^{2}$, L Sanchis-Sanchez ${ }^{2}$, P A Schneider ${ }^{4}$, A Snicker ${ }^{10}{ }^{\infty}$, \\ $P_{\text {Vallejos }}^{8}$ (1) and the AUG Team and the EUROfusion MST1 Team ${ }^{11}$ \\ ${ }^{1}$ CCFE, Culham Science Centre, Abingdon, Oxfordshire, United Kingdom \\ ${ }^{2}$ FAMN Department, Faculty of Physics, University of Seville, Seville, Spain \\ ${ }^{3}$ General Atomics, PO Box 85608, San Diego, United States of America \\ ${ }^{4}$ Max Planck Institute fur Plasmaphysik, Garching, Germany \\ ${ }^{5}$ FOM-Institute DIFFER, Nieuwegein, The Netherlands \\ ${ }^{6}$ Instituto de Plasmas e Fusão Nuclear, Instituto Superior Técnico, Portugal \\ ${ }^{7}$ Barcelona Supercomputing Center (BSC), Barcelona, Spain \\ ${ }^{8}$ VR/Royal Institute of Technology KTH, Sweden \\ ${ }^{9}$ ICREA, Barcelona, Spain \\ ${ }^{10}$ Aalto University, Aalto, Finland \\ E-mail: Sergei.Sharapov@ukaea.uk
}

Received 11 July 2017, revised 27 September 2017

Accepted for publication 4 October 2017

Published 2 November 2017

\begin{abstract}
Dedicated studies performed for toroidal Alfvén eigenmodes (TAEs) in ASDEX-Upgrade (AUG) discharges with monotonic $q$-profiles have shown that electron cyclotron resonance heating (ECRH) can make TAEs more unstable. In these AUG discharges, energetic ions driving TAEs were obtained by ion cyclotron resonance heating (ICRH). It was found that off-axis ECRH facilitated TAE instability, with TAEs appearing and disappearing on timescales of a few milliseconds when the ECRH power was switched on and off. On-axis ECRH had a much weaker effect on TAEs, and in AUG discharges performed with co- and counter-current electron cyclotron current drive (ECCD), the effects of ECCD were found to be similar to those of ECRH. Fast ion distributions produced by ICRH were computed with the PION and SELFO codes. A significant increase in $T_{\mathrm{e}}$ caused by ECRH applied off-axis is found to increase the fast ion slowing-down time and fast ion pressure causing a significant increase in the TAE drive by ICRH-accelerated ions. TAE stability calculations show that the rise in $T_{\mathrm{e}}$ causes also an increase in TAE radiative damping and thermal ion Landau damping, but to a lesser extent than the fast ion drive. As a result of the competition between larger drive and damping effects caused by ECRH, TAEs become more unstable. It is concluded, that although ECRH effects on AE stability in present-day experiments may be quite significant, they are determined by the changes in the plasma profiles and are not particularly ECRH specific.
\end{abstract}

Keywords: energetic particles, Alfvén eigenmodes, ECRH, ECCD, ICRH

(Some figures may appear in colour only in the online journal)

${ }^{11}$ H Meyer et al 2017 Nucl. Fusion 57102014. 


\section{Introduction}

Good confinement of energetic ions produced by neutral beam injection (NBI), ion cyclotron resonance heating (ICRH), and fusion-born alpha-particles during the slowingdown times is a crucial requirement for ITER plasma operations in burning plasma regimes [1,2]. Since the energetic particle populations on ITER are super-Alfvénic and their velocities $V_{\alpha}$ satisfy $V_{T_{\mathrm{i}}} \ll V_{\alpha} \approx V_{\mathrm{A}} \ll V_{T_{\mathrm{e}}}$, resonant interaction between energetic particles and weakly-damped Alfvén eigenmodes (AEs) may drive AEs unstable. If the amplitudes of AEs become high enough, a significant increase in the radial transport of the resonant energetic ions could affect significantly the power deposition profiles and increase fast ion loss to the first wall $[1,2]$.

Possible suppression/control of Alfvén instabilities using tools other than energetic particles is a potentially important new research avenue for burning plasmas, which started a decade ago from observation of mitigation of AEs with electron cyclotron resonance heating (ECRH) on the DIII-D tokamak [3]. Figure 1 shows the cross-section and safety factor $q(r)$ in the representative reconstructed equilibria of DIII-D with NBI and ECRH applied shot-byshot from the plasma centre (position P1) to outside the $q_{\text {min }}$ surface (position P5). Figure 2 shows that high amplitude multiple AEs were excited by NBI when 1.9 MW of ECRH was applied close to the magnetic axis, while the AE amplitudes and the number of unstable modes were much smaller when ECRH was applied at the position of $q_{\mathrm{min}}$.

The observed mitigation of AEs with ECRH is of great interest as ECRH is foreseen as one of the main heating and current drive techniques in ITER, and ECRH usually does not affect strongly fast ions. Two questions were addressed following the observations [3]: (i) is the ECRH suppression mechanism on AEs reproducible on other machines, and (ii) what is the physics behind this effect. To answer the first question, dedicated experiments were performed on the ASDEX-Upgrade (AUG) tokamak in 2014 [4]. A technique consisting of scanning ECRH positions $q_{\text {min }}$ (zero magnetic shear) region was employed, similar to that for DIII-D, and the ECRH mitigation effect on AEs was confirmed. Moreover, a complete suppression of AEs with ECRH applied at $q_{\text {min }}$ was achieved in these AUG experiments as figure 3 demonstrates.

A comparison between the TRANSP [5] calculation for classical slowing-down of beam ions and the FIDA [6] measurements of the beam ions in the two comparison discharges shows that a nearly classical beam distribution was obtained in the AUG discharge without AEs. In the discharge with AEs, a difference of up to $\sim 30 \%$ was seen for the fast ions in the plasma core as figure 4 shows.

The interpretation of the observed effect of ECRH mitigation of AEs requires a more specific information on the AEs mitigated. These modes associated with reversed-shear discharges were first investigated in detail on JET, where they were called Alfvén cascade (AC) eigenmodes [7, 8], and later became known as reversed-shear AEs on DIII-D [3]. In
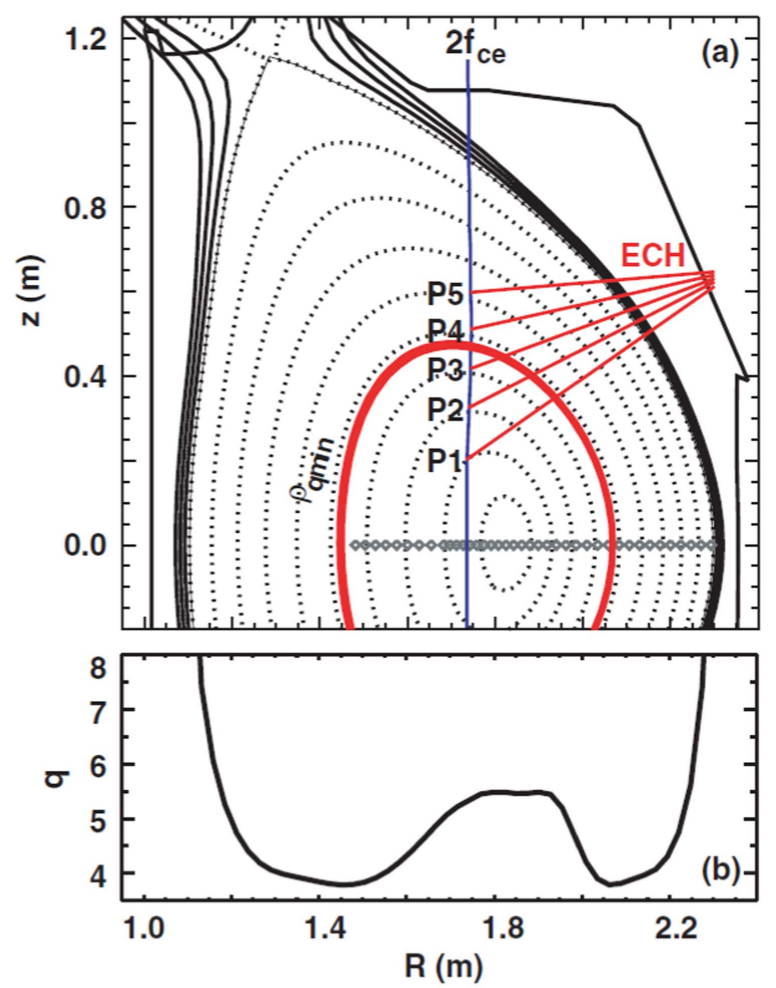

Figure 1. DIII-D equilibrium for reversed-shear discharges with NBI and ECRH applied at positions P1-P5. The position of $q_{\min }$ is shown in red. $B=2.0 \mathrm{~T}[3]$.

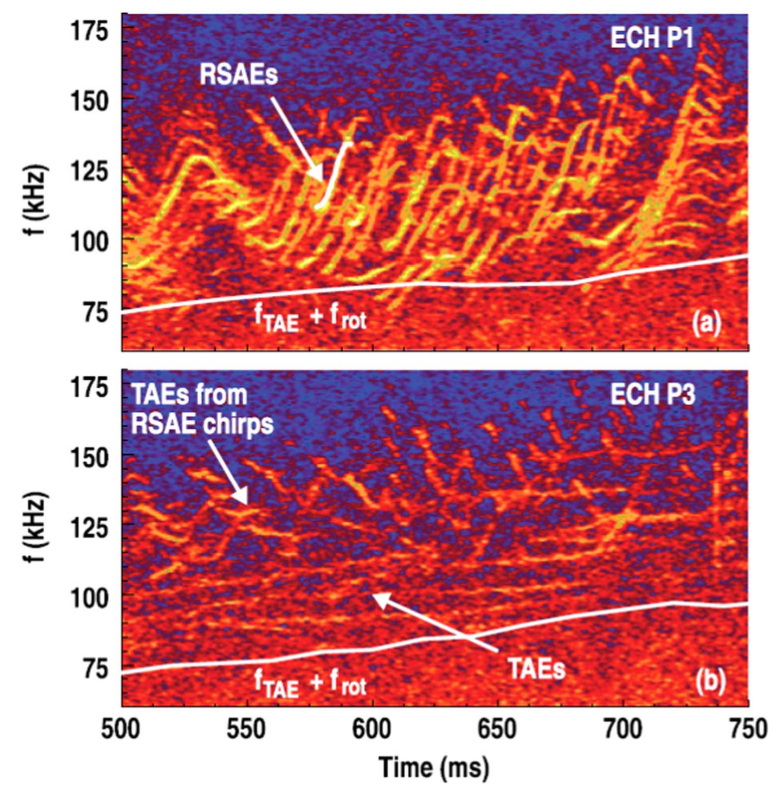

Figure 2. Top: interferometry data for density perturbation in AEs excited by NBI when $1.9 \mathrm{MW}$ of ECRH was applied at P1 position (DIII-D \#128564); bottom: AEs of much lower amplitudes and in less numbers are seen when ECRH is applied at P3 (\#128560) [3].

contrast to the well-known TAE modes [9], the ACs are associated with the existence of $q_{\min }$ away from the magnetic axis, are localised in the vicinity of $q_{\mathrm{min}}$, and their frequency $f_{\mathrm{AC}}$ tracks the evolution of $q_{\mathrm{min}}(t)$ in accordance with the 

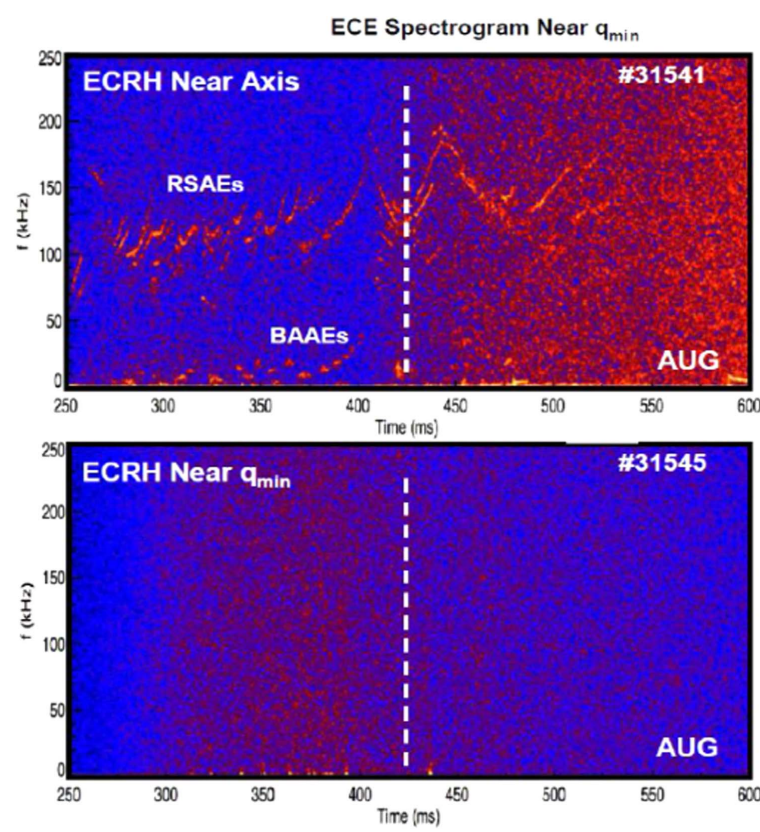

Figure 3. ECE data for $T_{\mathrm{e}}$ perturbations in AEs excited in two AUG discharges with ECRH applied near magnetic axis (top) and near $q_{\min }$ (bottom) [4]. The broken white line indicates time of $q_{\min }=$ integer when AEs with all mode numbers appear and could be driven.
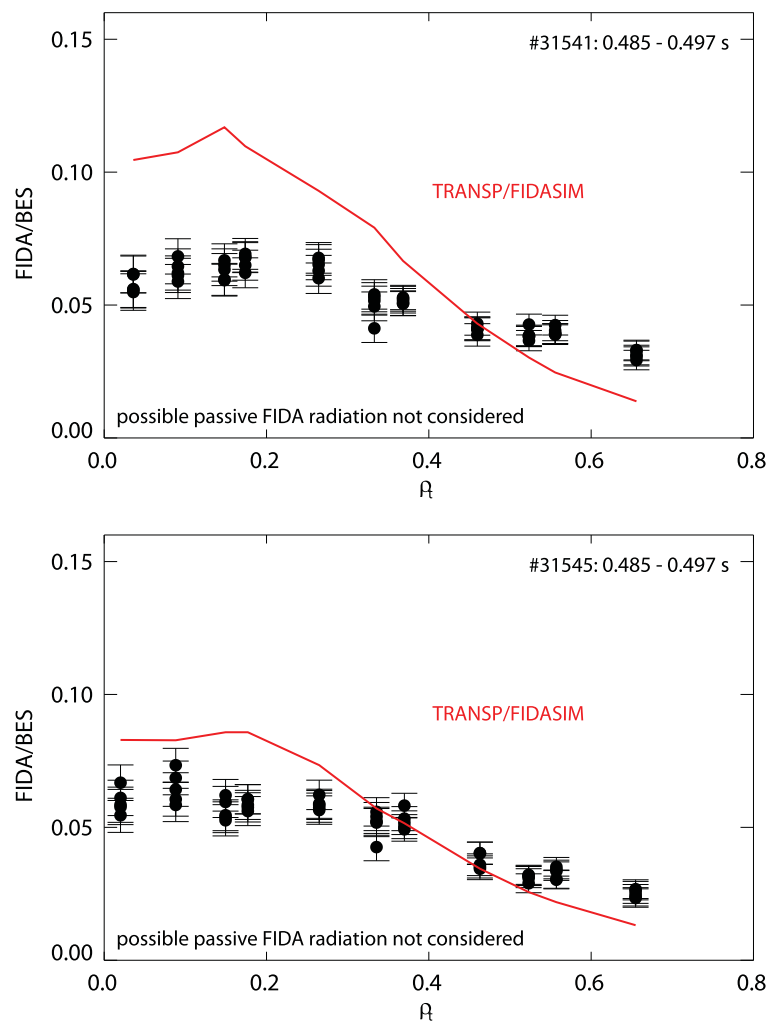

Figure 4. Radial profiles of NBI ions measured with the fast ion $D_{\alpha}$ (FIDA) diagnostics and computed with the TRANSP code in the two comparison discharges. Here, $\rho_{\mathrm{t}}=\left(\psi_{\mathrm{t}} / \psi_{\mathrm{t}}(a)\right)^{1 / 2}$ is radial coordinate associated with toroidal magnetic flux $\psi_{\mathrm{t}}$, and the vertical axis shows the ratio between the measured FIDA radiation intensity (in $\mathrm{Ph} / \mathrm{s} / \mathrm{Sr} / \mathrm{nm} / \mathrm{m}^{2}$ ) and the measured beam emission radiation (BES) intensity (same units). expression

$$
2 \pi f_{\mathrm{AC}}=\left|\frac{m}{q_{\min }(t)}-n\right| \cdot \frac{V_{\mathrm{A}}}{R_{0}}+\Delta f
$$

where $m, n$ are poloidal and toroidal mode numbers, $R_{0}$ is the major radius, and $\Delta f$ includes effects associated with thermal plasma pressure and pressure gradient. The frequencies of ACs lie in the band between the geodesic acoustic mode (GAM) and TAE frequencies,

$$
\begin{aligned}
f_{\mathrm{GAM}} & \cong \frac{1}{2 \pi}\left[\frac{2}{M_{\mathrm{i}} R_{0}^{2}}\left(T_{\mathrm{e}}+\frac{7}{4} T_{\mathrm{i}}\right)\right]^{1 / 2} \leqslant f_{\mathrm{AC}} \leqslant f_{\mathrm{TAE}} \\
& \cong \frac{V_{\mathrm{A}}}{4 \pi q R_{0}},
\end{aligned}
$$

where $M_{\mathrm{i}}$ is ion mass, $T_{\mathrm{e}}$ and $T_{\mathrm{i}}$ are electron and ion temperatures.

It was found as a result of additional dedicated experiments on DIII-D that with ECRH applied in the vicinity of $q_{\text {min }}$ the electron temperature increases up to the point when GAM frequency becomes comparable to the TAE frequency, which is rather low in DIII-D discharges with $q_{\text {min }} \sim 2-4$. In this way, the frequency band for AC existence shrinks, and $\mathrm{ACs}$ are suppressed due to higher ion Landau damping associated with the acoustic oscillations [10].

This effect of the shrinking mode frequency band cannot, however, be applied to TAEs, and so the search for $\mathrm{ECRH} /$ electron cyclotron current drive (ECCD) effects on TAEs requires consideration of some other possibilities. Early observations on DIII-D did indicate that ECRH could also interact with TAEs [11], but more extended data were required for investigating such possibility.

\section{The AUG experiment on ECRH effects on TAEs}

To study ECRH/ECCD effects on TAE, discharges with monotonic $q(r)$-profiles and TAEs existing for long time were developed on AUG. Since it is more difficult to excite TAEs with NBI in AUG flat top plasmas with high currents $I_{P}>500 \mathrm{kA}$, ICRH-accelerated ions were employed for TAE excitation. To use hydrogen minority ICRH on the magnetic axis meant that a magnetic field of $B=2.446 \mathrm{~T}$ was required, with plasma currents at the flat top of $I_{P}=0.8 \mathrm{MA}$. A correct choice of plasma density was one of the main issues in the experiment as the density should be neither too low (for avoiding supra-thermal electrons) nor too high (for facilitating ICRH tail of fast ions resonating with TAEs). The range of $n_{\mathrm{e}}(0) \approx 3-4.5 \times 10^{19} \mathrm{~m}^{-3}$ was chosen as an optimal one. For the ICRH power levels required, the AUG discharges were in the $\mathrm{H}$-mode, and the additional use of ECRH increased plasma density in these AUG discharges by $\approx 20 \%$, in a broad agreement with the interplay between ECRH and plasma density described in [12]. 


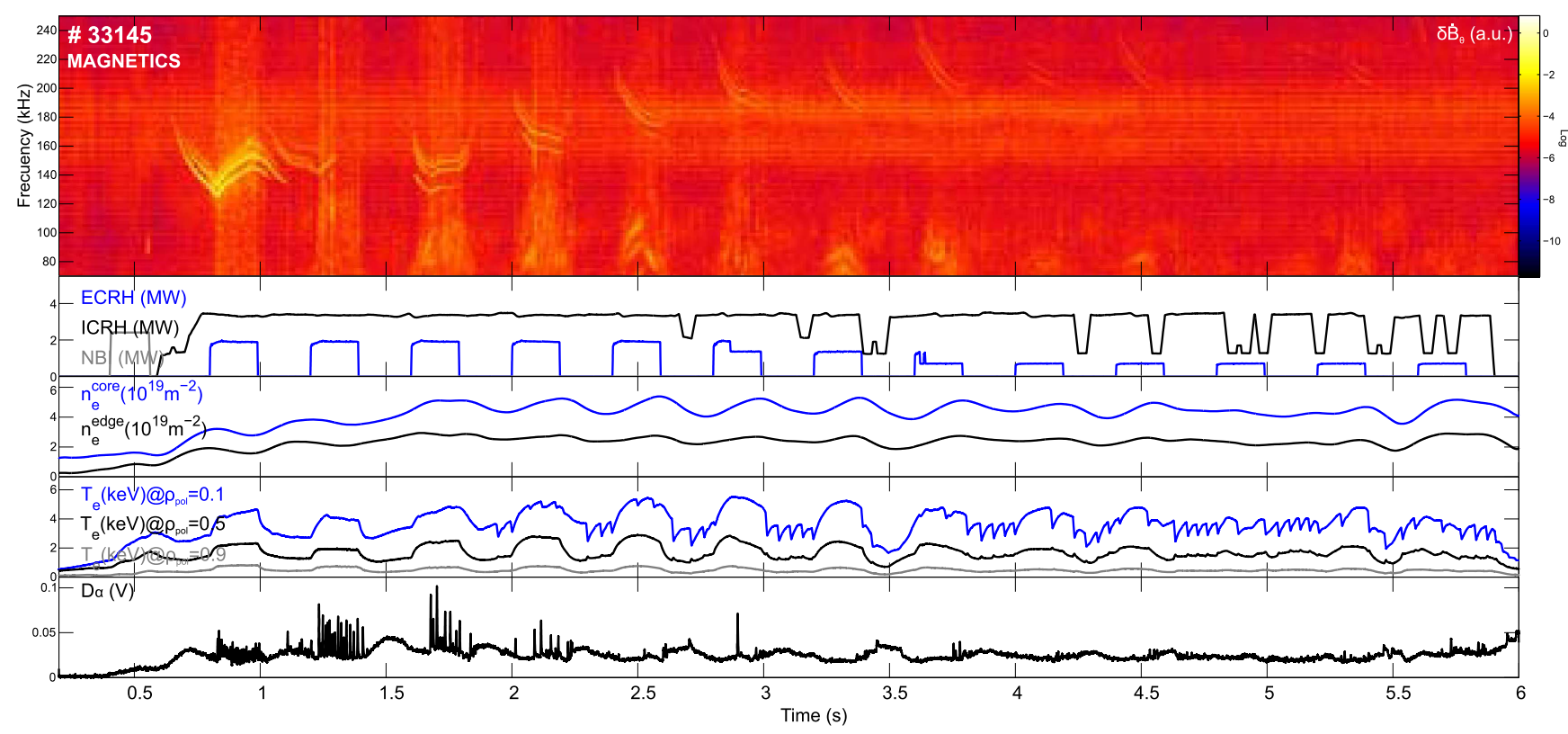

Figure 5. From top to bottom: (i) magnetic spectrogram for perturbed magnetic field measured with Mirnov sensors showing excitation of TAEs; (ii) power wave-forms of ICRH, ECRH and NBI; (iii) temporal evolution of $n_{\mathrm{e}}$ on-axis and at the plasma edge, (iv) temporal evolution of $T_{\mathrm{e}}$ on-axis, at half-radius, and at the plasma edge, and (v) $D_{\alpha}$ signal. AUG discharge \#33145 with ECRH at $\rho_{p} \approx 0.3$.

\subsection{Facilitation of TAE instability with ECRH applied off-axis}

Figure 5 shows typical observation of ICRH-driven TAEs in the dedicated AUG discharge with ECRH modulated power $200 \mathrm{~ms}$ on $/ 200 \mathrm{~ms}$ off applied at normalised radius $\rho_{p} \approx 0.3$. Here, $\rho_{p}=(\psi / \psi(a))^{1 / 2}$ is a radial coordinate determined by the poloidal flux $\psi$. TAEs are excited throughout the whole discharge from $\sim 0.5$ to $\sim 5.5 \mathrm{~s}$. Significant changes in TAEs are seen in correlation with the ECRH modulation, together with large changes in plasma density and temperature. Figure 6 shows that TAEs with toroidal mode numbers from $n=2$ to $n=6$ were excited in the frequency range $125 \mathrm{kHz}<f_{\mathrm{TAE}}<230 \mathrm{kHz}$. The frequency sweeping of TAEs is associated with the density increase during the ECRH and the corresponding decrease in Alfvén velocity $V_{\mathrm{A}} \propto B / n^{1 / 2}$. Finally, figure 7 shows that TAEs become unstable when ECRH is applied off-axis, and disappear after ECRH is switched off. A remarkably short time scale of few $\mathrm{ms}$ is observed in the correlation between ECRH on/off and TAE appearance/disappearance. A similar effect on TAEs was observed with ECRH applied at larger radius of $\rho_{p} \approx 0.5$.

Internal measurements of the TAE mode structure were performed in the AUG discharges with reflectometry [13] and ECE imaging (ECEI) [14] diagnostics covering the midradius plasma region as figure 8 shows. For TAE observed in discharge \#33145 at $\sim 1 \mathrm{~s}$, the ECEI measurements showed that the TAE mode structure is peaked at $R \sim 1.85-1.9 \mathrm{~m}$ corresponding to the mid-radius, in a robust agreement with TAE in figure 8 computed with incompressible ideal MHD spectral code MISHKA-1 [15].

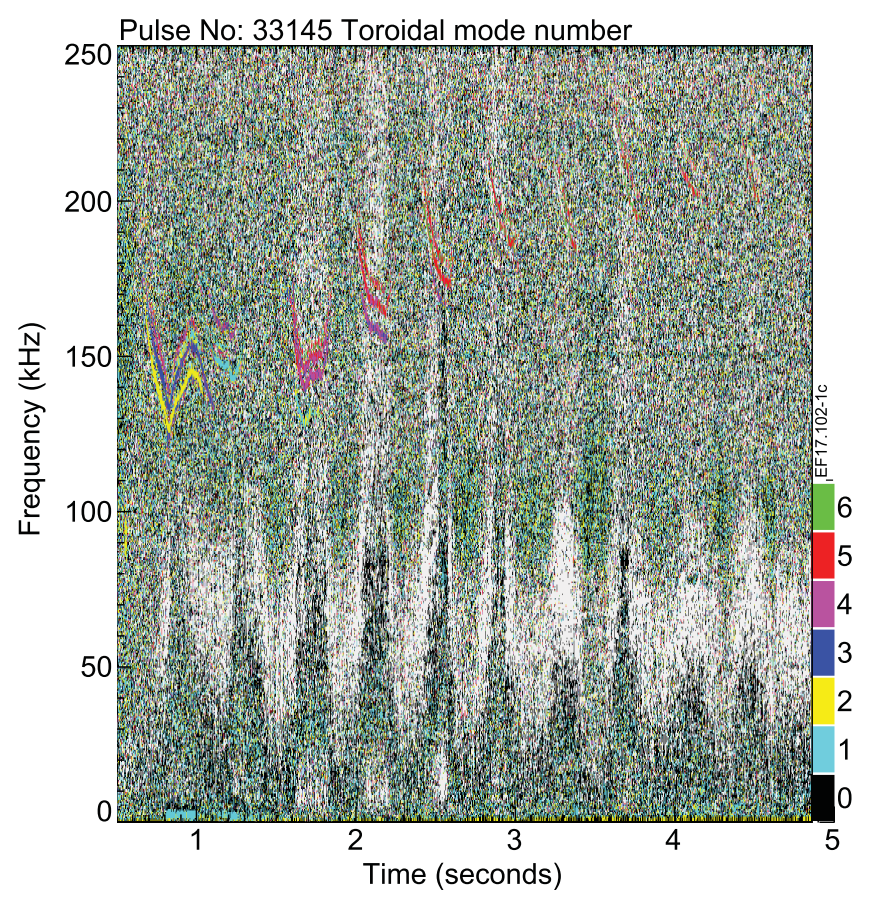

Figure 6. Phase magnetic spectrogram showing colour-coded toroidal mode numbers of the excited TAEs in figure 5.

\subsection{No effect on TAE stability from ECRH applied on-axis}

In contrast to discharges with ECRH applied off-axis, no effect on TAE stability was observed when ECRH was applied on-axis. Figure 9 shows magnetic spectrogram and temporal evolution of main plasma parameters in discharge \#33147 with on-axis ECRH. TAEs are observed at the 


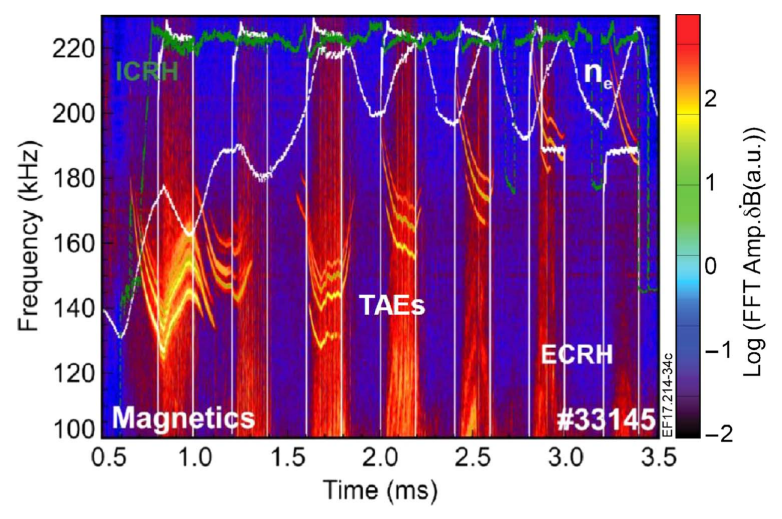

Figure 7. Zoom showing fine time scales for TAE excitation/ disappearance with ECRH.

beginning of this discharge only, at $t<1.3 \mathrm{~s}$, before the start of the modulation power wave-form of ECRH.

\subsection{Co- and counter-current ECCD applied at $\rho_{p} \approx 0.6$ affect TAE frequency, but not stability}

Taking into account recent demonstration of strong ECCD effects on beam-driven AEs in stellarators [16-18], a study of the effect of ECCD on TAEs has started on AUG. Although an unambiguous interpretation of the ECCD effects on AEs in stellarators is not accomplished yet, changes in the iota profile could be the main mechanism affecting AEs [18]. On AUG, four discharges were performed with co-current ECCD applied on-axis, at $\rho_{p}=0.3$, and $\rho_{p}=0.6$, with ECRH modulation rates $200 \mathrm{~ms} / 200 \mathrm{~ms}$ and $21 \mathrm{~ms} / 21 \mathrm{~ms}$. In the cases with short-period ECCD modulation, sawteeth did not appear in the discharges. One discharge (pulse \#33170) was performed with counter-current ECCD applied at $\rho_{p}=0.6$ with $21 \mathrm{~ms} / 21 \mathrm{~ms}$ modulation rate. The power of ECCD was deposited at the radius where the TAE mode structure was expected to be peaked, in order to maximise the effect on magnetic shear and the mode continuum damping. However, in all cases, ECCD was found to cause a modulation of the TAE frequency due to the plasma density variation as in the ECRH case, but no obvious effect on TAE stability was observed. Figure 10 shows a typical discharge with co-current ECCD applied at $\rho_{p}=0.6$. No significant difference is seen between ECCD and ECRH effects on TAEs, but more data are needed for a conclusion on the ECCD effects.

\section{Modelling of the AUG discharges}

To explain the observed evolution of TAEs during ECRH/ ECCD on AUG, the following effects were considered:

(i) Supra-thermal electrons excite TAEs (see, e.g. [19, 20]).

(ii) ECRH-driven energetic ions excite TAEs (see, e.g. [21, 22]).

(iii) The significant increase of $T_{\mathrm{e}}$ affects significantly both TAE damping (radiative, ion and electron Landau) and drive from ICRH ions (as slowing-down time increases). (iv) The density rise during ECRH shifts $V_{\mathrm{A}}$ into the waveparticle resonance region.

(v) ECRH/ECCD modifies $q$-profile affecting continuum damping of TAE.

ECE data were investigated to answer the question on the existence of significant population of supra-thermal electrons in these discharges. No evidence was found for supra-thermal electrons. The density range of $3-4.5 \times 10^{19} \mathrm{~m}^{-3}$ used in our discharges was too high for the supra-thermal electrons to appear, most typical densities for supra-thermal electrons on AUG are $\sim 2 \times 10^{19} \mathrm{~m}^{-3}$.

Next, the possibility of ECRH-generated fast ions was investigated from the neutral particle analyser [23] measurements. No evidence was found for an ECRH-driven fast ion population in our discharges.

With the first two effects not playing a role, the problem of explaining the observed TAE evolution was reduced to that of computing accurately the competition between TAE drive and TAE damping in our discharges. For computing the ICRH-drive for TAE, modelling of ICRH tail was performed with the PION code [24] that provides a simplified, but fast and reliable, assessment of the power deposition profile and temperature of the ICRH-accelerated ions, and the SELFO code [25] that computes the distribution function with more physics included, such as the coupling between the fast ions and the global wave-field in the ion cyclotron frequency range launched by ICRH antenna. These results were followed by a drive assessment with the CASTOR-K code [26] that computes the mode, the fast ion orbits, and the linear wave-toparticle power transfer, the initial value nonlinear particlefollowing HAGIS code [27], and the linear gyro-kinetic LIGKA code [28]. To compute TAE damping effects, the LIGKA code was employed, which provides a description of background kinetic and fast particle effects on the MHD stability in tokamaks.

\subsection{ICRH modelling}

Three comparison discharges, \#33147 (on-axis ECRH), \#33150 (ECRH @ $\rho_{p}=0.3$ ), and \#33151 (ECRH higher power @ $\rho_{p}=0.5$ ) were analysed with the PION and SELFO codes. A significant ECRH effect on the fast ion energy content was found to be caused by the increase in slowingdown time of the fast ions. The cases with TAEs close to the marginal stability were of major interest, with an example of TAEs with $n=5$ and $n=6$ excited in AUG discharge \#33150 at $2.4 \mathrm{~s}$. The measured plasma parameters and SELFO computed fast ion energy contents are shown in figures 11 and 12 for this AUG discharge of interest. The fast ion energy content increases by $40 \%$ with ECRH applied offaxis as fast $\mathrm{H}$ slowing-down time more than doubles from $\sim 0.07 \mathrm{~s}$ (at $2.25 \mathrm{~s}$ ) to $\sim 0.16 \mathrm{~s}$ (at $2.45 \mathrm{~s}$ ) as figure 12 shows.

It is seen from the SELFO modelling that the H-minority energy contents nearly doubles when ECRH is applied at $\sim 2.4 \mathrm{~s}$ and TAEs are excited. Figures 13 and 14 show the energy distribution functions and the radial fast ion pressure profiles for the near-threshold TAE-case in \#33150 at around 

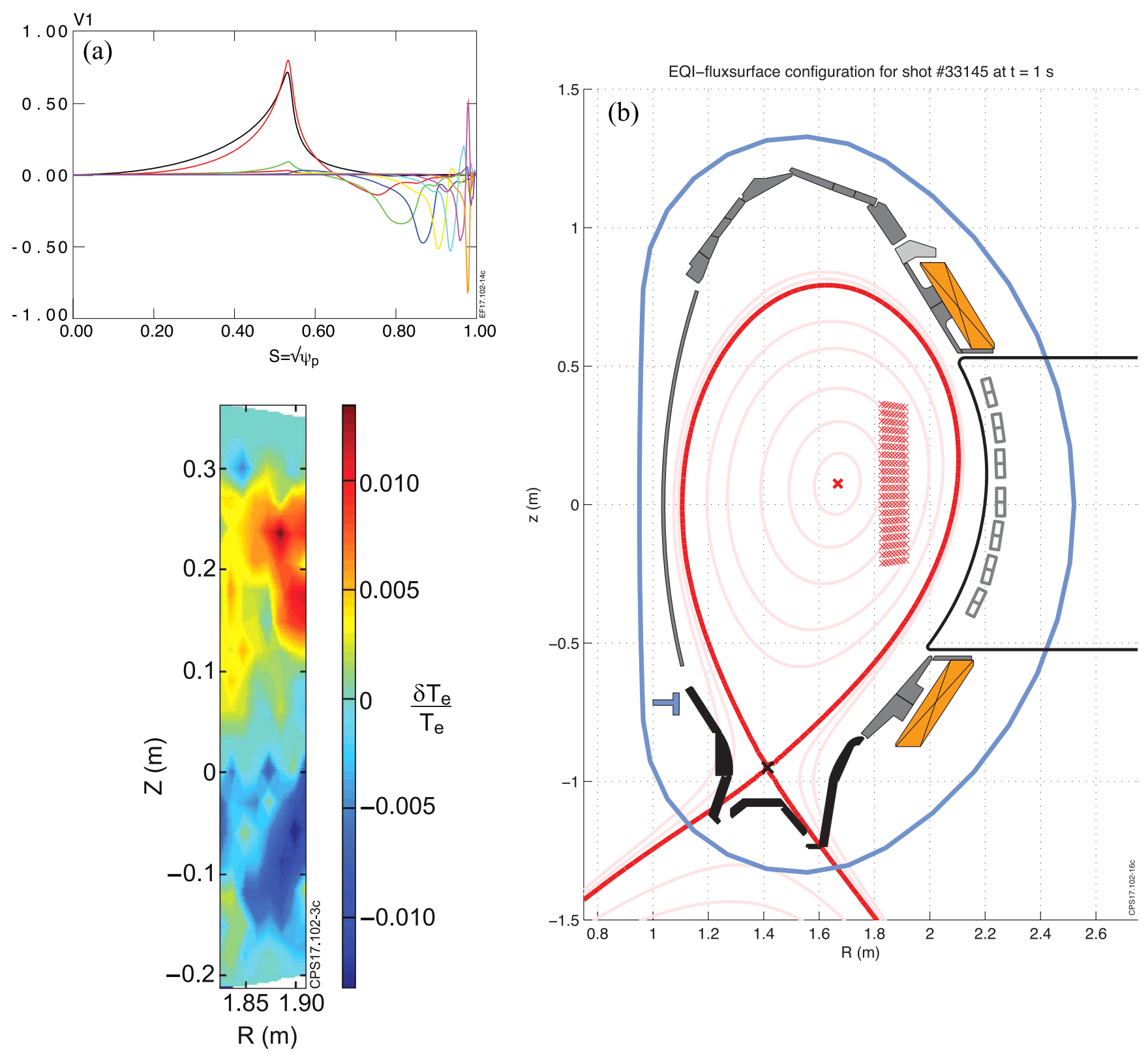

Figure 8. (a) Top: TAE mode structure computed with the MISHKA code [15], $V 1=S V_{\mathrm{r}}$ is radial velocity of the plasma displacement; bottom: amplitude of the TAE as function of $R, Z$ measured with ECEI array. Here, $R=1.85 \mathrm{~m}$ corresponds to $s \equiv \rho_{p} \approx 0.44$, and $R=1.9$ m corresponds to $s \equiv \rho_{p} \approx 0.55$. (b) Position of the ECEI imaging array measuring $T_{\mathrm{e}}$ perturbations in the AUG experiments on ECRH/TAE.

$\sim 2.4 \mathrm{~s}$. It is seen that the fast ion energy assessed at $2.34 \mathrm{~s}$ first increases at $2.51 \mathrm{~s}$, and then decreases at $2.59 \mathrm{~s}$ in agreement with the dynamics of the slowing-down time shown in figure 12 .

For comparing the on-axis and off-axis ECRH effects, it was found that the ECRH effect on the shape of $T_{\mathrm{e}}$ profile makes slowing-down time $\tau_{\mathrm{SD}}(0)$ in the ECRH on-axis discharge higher than $\tau_{\mathrm{SD}}(0)$ in the discharges with ECRH offaxis. However, $\tau_{\mathrm{SD}}\left(\rho_{p}=0.4\right)$ in the case of ECRH on-axis is significantly lower than that in the discharges with ECRH offaxis. As a result; the increase of the slowing-down time affects significantly fast ions in the ECRH off-axis discharges, and less so-in the ECRH on-axis discharges.

\subsection{Modelling of TAE drive by ICRH-accelerated ions}

For the modelling of TAE drive by ICRH-accelerate ions, codes CASTOR-K, HAGIS, and LIGKA were used. Figure 15 shows plasma density, temperature, and $q(r)$ profiles just before ECRH and just after ECRH in the discharge \#33150. Deuterium plasma is considered with $Z_{\text {eff }}=2$, with carbon impurity of $n_{\mathrm{c}} / n_{\mathrm{e}}=4 \%$ and tungsten impurity of $n_{\mathrm{w}} / n_{\mathrm{e}}=0.05 \%$. Figure 16 shows TAEs and Alfvén continua with $n=5$ and 6 computed with the LIGKA code corresponding to TAEs observed in AUG discharge \#33150 at the near-threshold time $\sim 2.4 \mathrm{~s}$.

To assess the fast ion phase space, where the main contribution comes from the wave-particle interaction, 


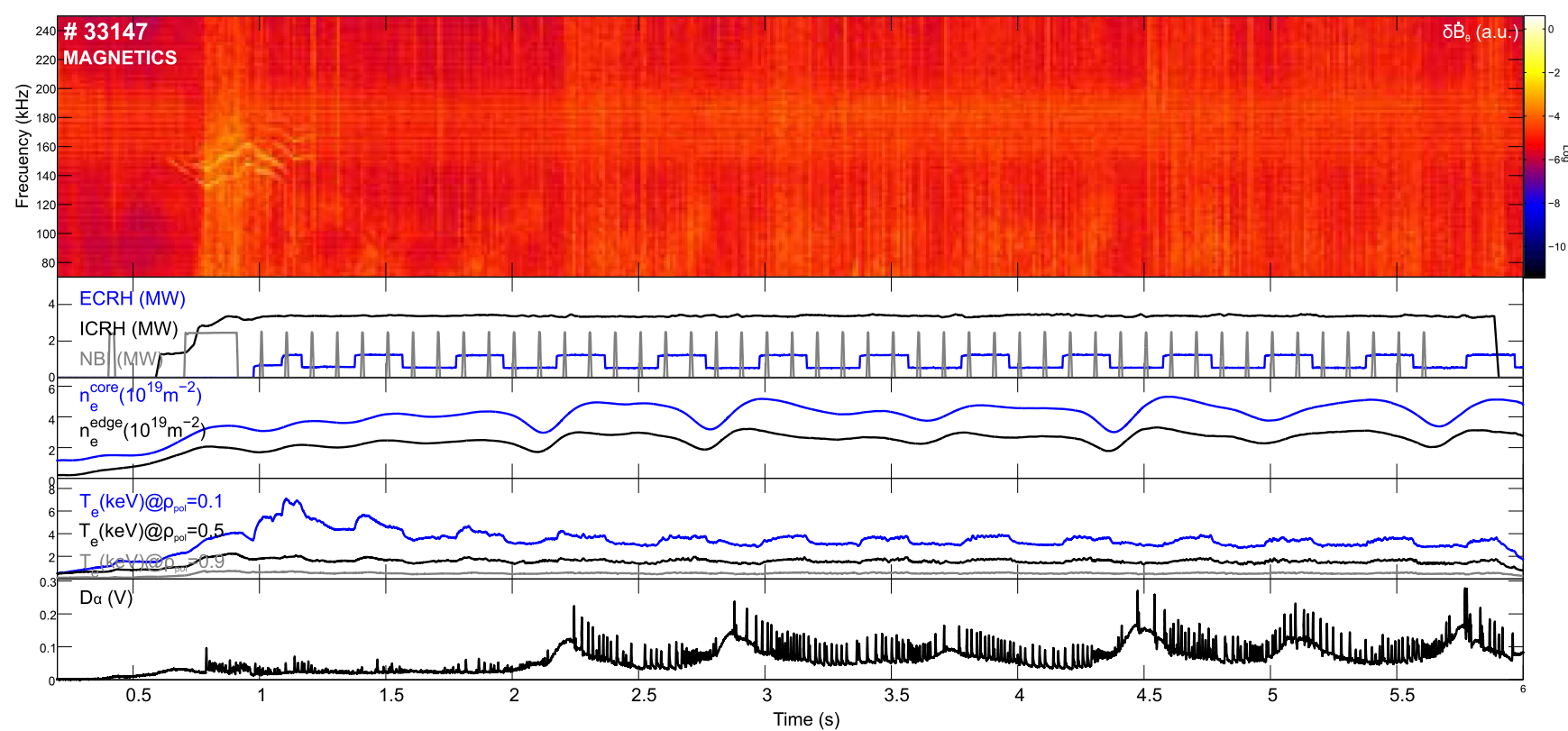

Figure 9. From top to bottom: (i) magnetic spectrogram showing excitation of TAEs; (ii) power wave-forms of ICRH, ECRH, and NBI; (iii) temporal evolution of $n_{\mathrm{e}}$ on-axis and at the plasma edge, (iv) temporal evolution of $T_{\mathrm{e}}$ on-axis, at half-radius, and at the plasma edge, and (v) $D_{\alpha}$ signal. AUG discharge \#33147 with on-axis ECRH.

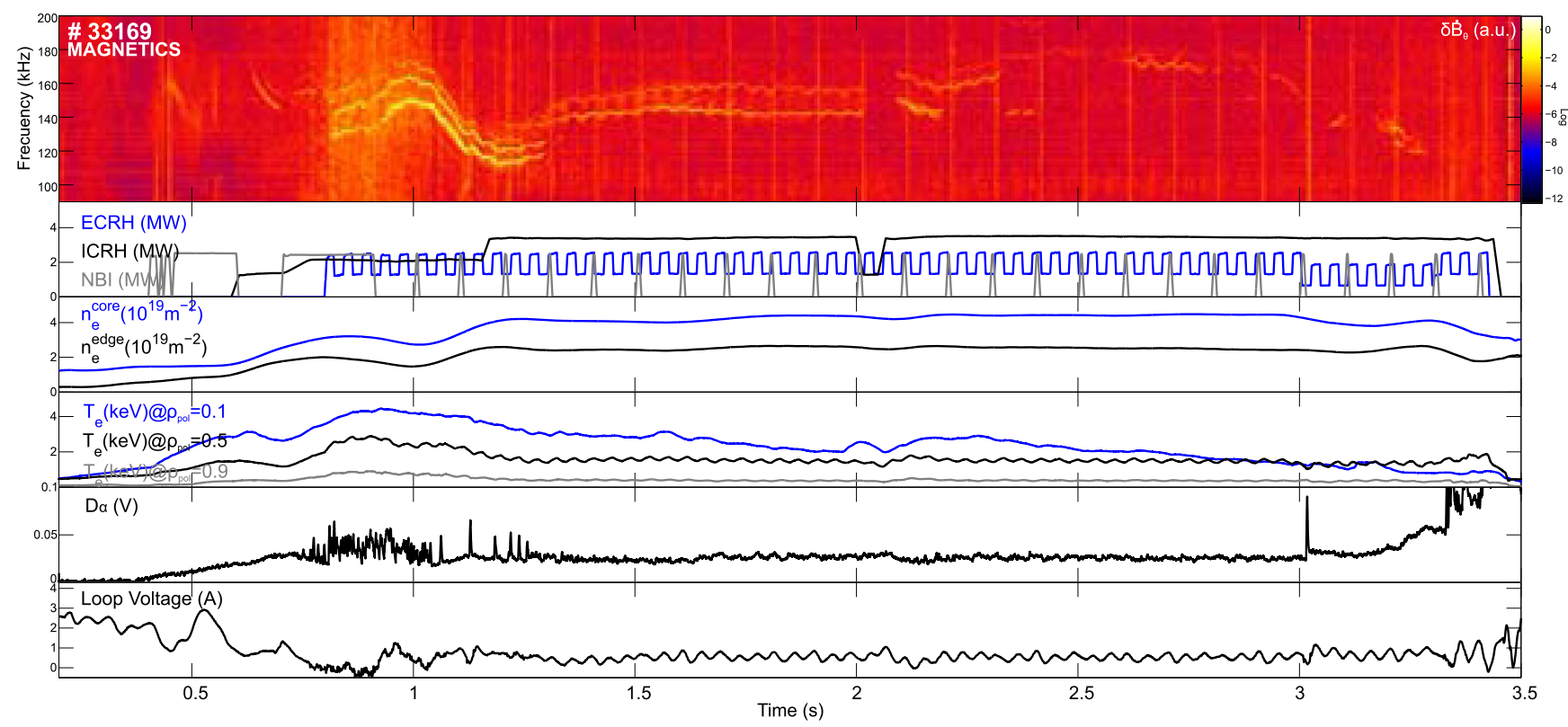

Figure 10. From top to bottom: (i) magnetic spectrogram showing excitation of TAEs; (ii) power wave-forms of ICRH, ECRH, and NBI; (iii) temporal evolution of $n_{\mathrm{e}}$ on-axis and at the plasma edge, (iv) temporal evolution of $T_{\mathrm{e}}$ on-axis, at half-radius, and at the plasma edge, and (v) $D_{\alpha}$ signal, (vi) loop voltage. AUG discharge \#33169 with co-current ECCD applied at $\rho_{p} \approx 0.6$.

resonance map of the contours of

$$
\Omega \equiv \omega-n \omega_{\phi}-p \omega_{\vartheta}=0
$$

was computed with the HAGIS and CASTOR-K codes for the on-axis ICRH with the trapped fast ion banana orbits having their turning points at $R=R_{0}$. Here, $\omega_{\vartheta}=\omega_{\vartheta}\left(E, P_{\varphi}, \Lambda,\right)$ and $\omega_{\varphi}=\omega_{\varphi}\left(E, P_{\varphi}, \Lambda\right)$ are the poloidal orbit frequency and the toroidal precession frequency of the fast ions, $n$ and $\omega$ are toroidal mode number and frequency of the mode, $p$ is the bounce harmonic, and $E=m V^{2} / 2, P_{\varphi}=e_{\mathrm{i}} \psi_{p}+m_{\mathrm{i}} R V_{\|}\left(B_{\varphi} / B\right), \Lambda=$ $\mu B_{0} / E$ are the phase space variables describing energy, toroidal canonical momentum, and pitch-angle of the fast ion orbits. The banana orbits have zero parallel velocity at their turning points, and so the toroidal canonical momentum depends on the poloidal flux $\psi_{p}$ only at these points, $P_{\varphi}=e_{\mathrm{i}} \psi_{p}(Z)$, where $e_{\mathrm{i}}$ is the fast ion charge. Next, the ions accelerated by on-axis ICRH, have their banana orbit tips at the magnetic axis, $R=R_{0}$, for which $\Lambda=1$. Therefore, in the case of on-axis ICRH, the structure of the resonances in equation (3) depends on two variables only, $E, P_{\varphi}$ (for fixed $\Lambda=1$ ), and the canonical 


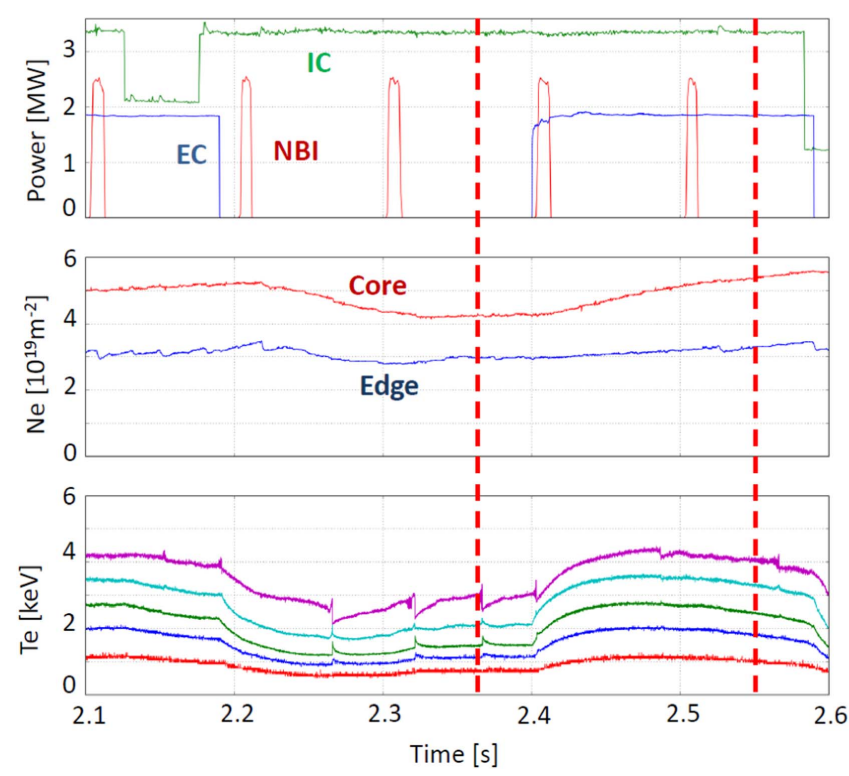

Figure 11. Top: power wave-forms of ICRH, ECRH, and NBI; middle: plasma density evolution in the plasma core and at the edge, and bottom: $T_{\mathrm{e}}$ evolution at different radii. The time window for the ICRH modelling is shown with two vertical broken lines.

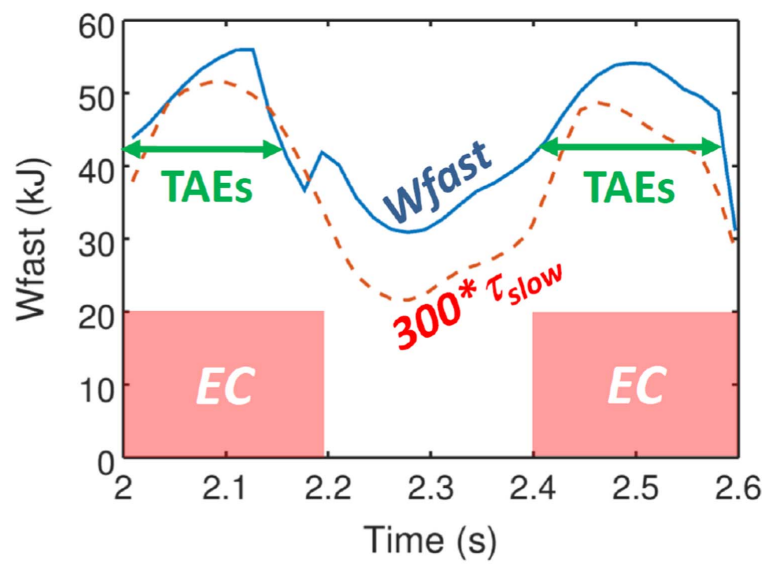

Figure 12. Temporal evolution of the fast ion energy content (blue line) in AUG pulse \#33150 computed with the SELFO code.

Temporal evolution of the slowing-down time of ICRH-accelerated hydrogen ions is also shown in red broken line.

variable could be substituted by $Z=Z\left(P_{\varphi}\right)$. A $2 \mathrm{D}$ plot of the resonances could be made then in the form $1 / \Omega(E, Z)$ by launching ions in the range of energies and radii of interest, computing for every ion launched $\omega_{\vartheta}, \omega_{\varphi}$, and building a 2D matrix representing equation (3). Figure 17 shows the resulting plot for the $n=5 \mathrm{TAE}$, from which one could identify the regions of phase space where a resonant interaction governed by equation (3) could occur. It is seen that the strongest resonance with $p=0$ corresponds to ICRH-accelerated trapped ions in the energy range between 200 and $500 \mathrm{keV}$. Since no significant resonance interaction is found at higher energies of the ions, an increase in driving the TAEs could not be achieved by generation of yet higher energy tail (e.g. via an increase in ICRH power increase or a decrease in $\mathrm{H}$ concentration). An assessment of the

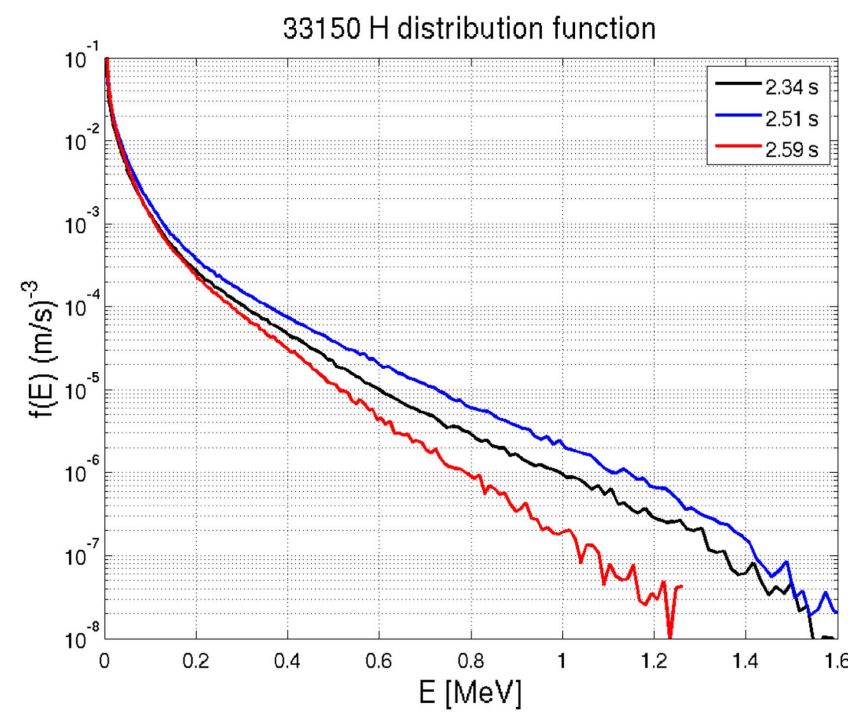

Figure 13. Energy distribution functions at different times ions computed with SELFO for fast $\mathrm{H}$ ions in \#33150.

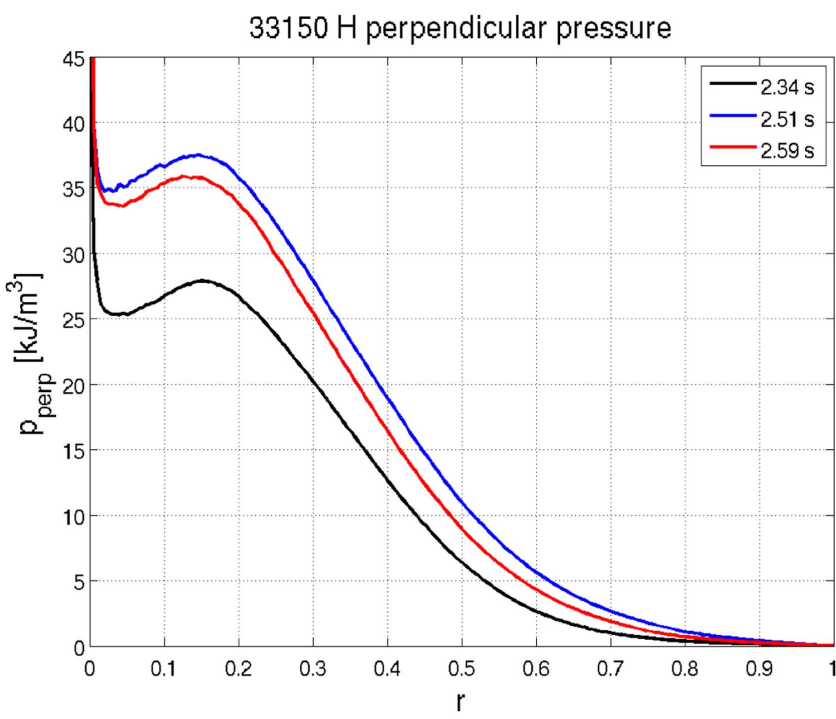

Figure 14. Profiles of perpendicular pressure of fast $\mathrm{H}$ ions computed with SELFO.

temporal evolution of the fast ion distribution following the applied ECRH off-axis shows an increase in the TAE growth rate by up to an order of magnitude depending on the TAE mode structure.

\subsection{Kinetic LIGKA modelling of TAE damping effects during $E C R H$}

Three main TAE damping mechanisms were investigated for the near-threshold TAE case in AUG discharge \#3315:

(i) Continuum damping.

(ii) Thermal ion Landau damping.

(iii) Radiative damping.

The electron damping effects were found to be small. For the continuum damping, no significant change in the distance between TAE localisation and the continuum crossing point 

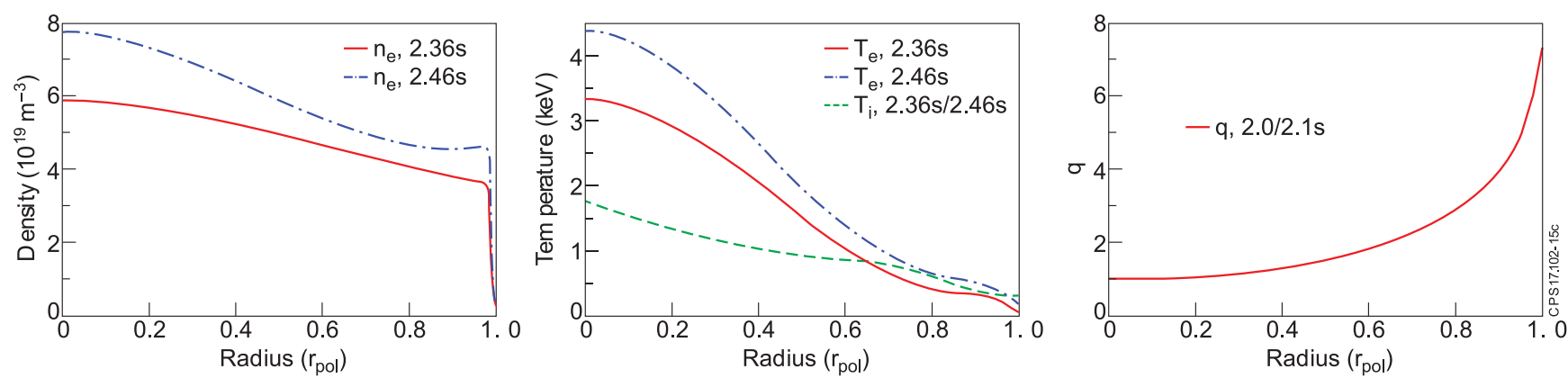

Figure 15. Left: $n_{\mathrm{e}}(r)$ just before ECRH, $t \sim 2.36 \mathrm{~s}$ (no TAE), and just after ECRH, $t \sim 2.46 \mathrm{~s}$ (TAEs excited); middle: $T_{\mathrm{i}}(r)$ measured in \#33150. Also $T_{\mathrm{e}}(r)$ are shown at $t \sim 2.36 \mathrm{~s}$ and $t \sim 2.46 \mathrm{~s}$; right: $q(r)$-profile used in the modelling.

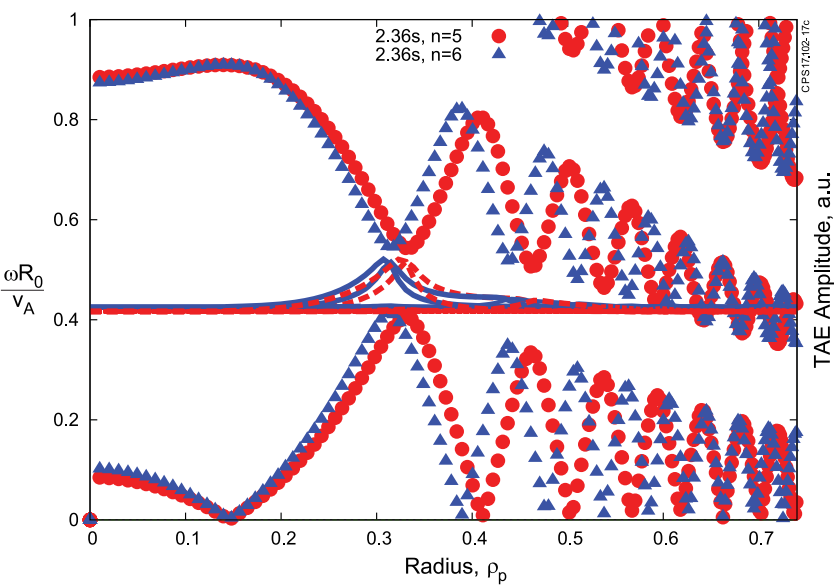

Figure 16. Core-localised TAEs with $n=5$ (solid blue line) and $n=6$ (broken red line) computed with the LIGKA code. Alfvén continua for $n=5,6$ are shown with red circles and blue triangles respectively.

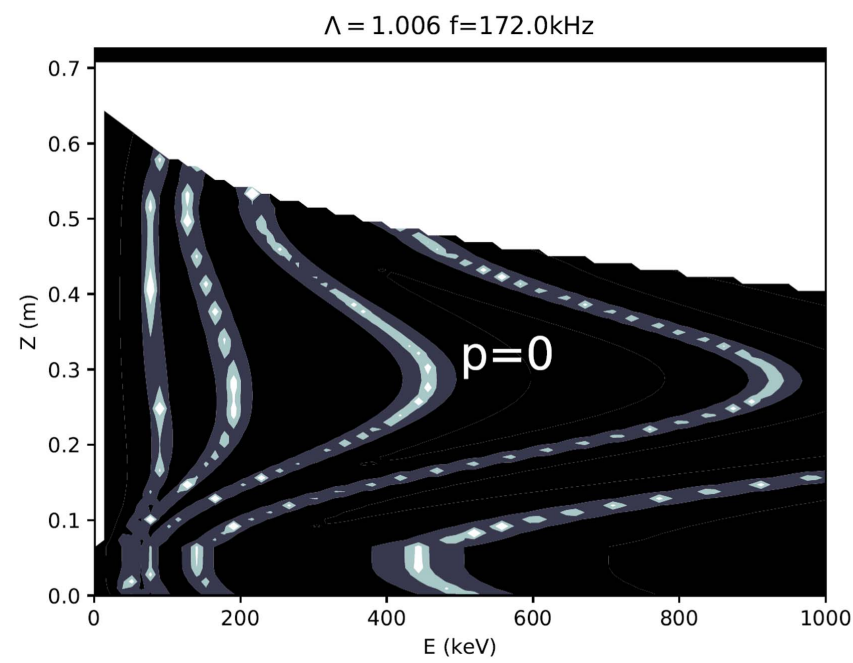

Figure 17. Resonance map for ICRH-driven TAEs with $n=5$ in AUG discharge \#33150, $t \sim 2.4 \mathrm{~s}$. The resonance lines are shown in white for $p=0, \pm 1$, and the most efficient resonance line with $p=0$ is marked.

was found during the ECRH phase. Hence, the role of the continuum damping for the given plasmas profiles (figure 15) was found to be negligible.

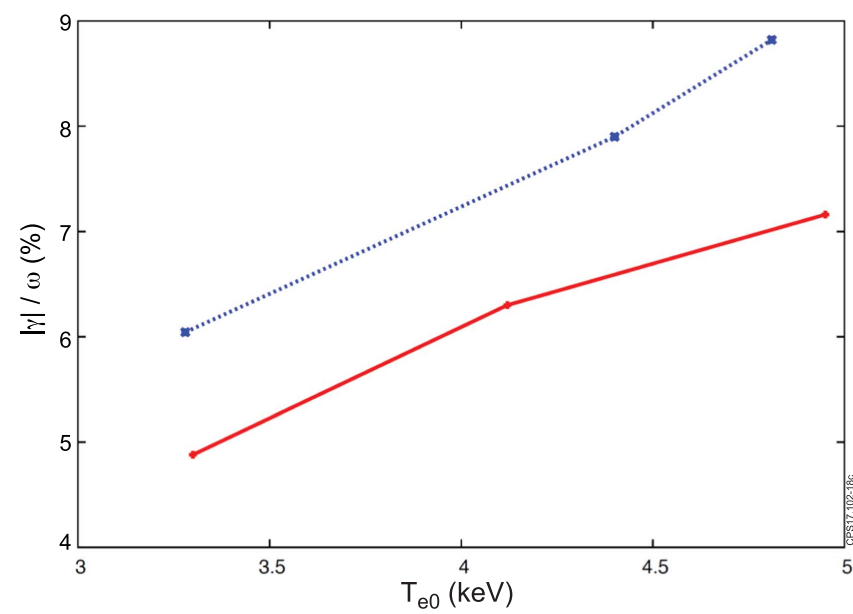

Figure 18. LIGKA: TAE damping at $t=2.36 \mathrm{~s}$ (red solid line) and $t=2.46 \mathrm{~s}$ (blue dotted line) in the $T_{\mathrm{e}}$ range in \#33150. With ECRH applied at $\rho_{p} \approx 0.3$, both ion Landau damping and radiative damping $\propto(3 / 4) T_{\mathrm{i}}+T_{\mathrm{e}}$ increase as shown by the modelling.

The remaining ion Landau and radiative damping effects were both found to increase significantly as $T_{\mathrm{e}}$ rises from the applied ECRH. Figure 18 summarises the LIGKA scan over $T_{\mathrm{e}}$ showing an increase in the damping rate of up to $\sim 50 \%$. However, the increased damping of TAEs is overcome by the additional ICRH drive due to higher $W_{\text {fast }}$.

\section{Conclusions}

In summary, following the discovery of the effects of ECRH on two types of Alfvén eigenmodes, ACs and TAEs on DIIID [3, 10, 11], dedicated AUG experiments were performed to investigate similar effects further. Only off-axis ECRH affects the mode stability, while ECRH on-axis had a relatively minor effect on TAE frequency only. It was established in DIII-D experiments $[3,10,11]$ that ECRH mitigates ACs in shear-reversed discharges due to the increasing $T_{\mathrm{e}}$ and $\nabla T_{\mathrm{e}}$ shrinking the AC frequency zone and causing high AC damping. In the case of monotonic $q(r)$-profiles, off-axis ECRH was found to facilitate TAE instability on DIII-D and in dedicated AUG experiments. A significant increase in fast 
ion population due to the increased slowing-down time was identified as the main effect in the case of TAEs.

We conclude that although ECRH effects on AE stability in present-day experiments may be quite significant, they are determined by the changes in the plasma profiles and are not particularly ECRH specific. This conclusion may be re-considered for the case of ECCD, but further investigation of ECCD effects on AEs are required to construct a statistically representative data base for tokamaks. The results on ECCD effects on AEs obtained on stellarators [16-18] are very encouraging and may further improve our understanding on what main physics effects one should explore for enhancing the ECCD effects on AEs.

In the case of ITER, the effects of ECRH/ECCD may be of importance for affecting ICRH- or NBI-driven AEs along the ITER transport path from the start of the plasma to the $Q=10$ scenario. On the path to the $Q=10$ operation, electron heating by fusion-born $\alpha$-particles may be not strong enough yet, so that ECRH/ECCD could play a role. In addition, possible effects on AEs from ECCD applied on ITER for stabilising sawteeth or NTMs could become an issue that needs a dedicated study to be performed.

\section{Acknowledgments}

This work has been carried out within the framework of the EUROfusion Consortium and has received funding from the Euratom research and training programme 2014-2018 under grant agreement No. 633053 and from the RCUK Energy Programme [grant number EP/P012450/1]. The views and opinions expressed herein do not necessarily reflect those of the European Commission.

\section{ORCID iDs}

S E Sharapov (1D https://orcid.org/0000-0001-7006-4876

J Galdon-Quiroga (i) https://orcid.org/0000-0002-7415-1894
A Snicker (ib https://orcid.org/0000-0001-9604-9666

P Vallejos (ib) https://orcid.org/0000-0003-4343-6325

\section{References}

[1] ITER Physics Basis 1999 Chapter 5 Nucl. Fusion 392471

[2] Fasoli A et al 2007 Chapter 5 Nucl. Fusion 47 S264

[3] Van Zeeland M A et al 2008 Plasma Phys. Control. Fusion 50 035009

[4] Garcia-Munoz M et al 2015 IAEA TM on Energetic Particles in Magnetic Confinement Systems (4-7 September (Vienna: IAEA)

[5] Hawryluk R J 1980 An empirical approach to tokamak transport Physics of Plasmas Close to Thermonuclear Conditions ed B Coppi et al vol 1 (Brussels: CEC) pp 19-46

[6] Geiger B et al 2013 Rev. Sci. Instrum. 84113502

[7] Berk H L et al 2001 Phys. Rev. Lett. 87185002

[8] Sharapov S E et al 2002 Phys. Plasmas 92027

[9] Cheng C Z, Chen L and Chance M S 1985 Ann. Phys. 16121

[10] Van Zeeland M A et al 2016 Nucl. Fusion 56112007

[11] Van Zeeland M A et al 2009 Nucl. Fusion 49065003

[12] Mlynek A et al 2011 Nucl. Fusion 51043002

[13] Cupido L et al 2006 Rev. Sci. Instrum. 77 10E915

[14] Freethy S J et al 2016 Rev. Sci. Instrum. 87 11E102

[15] Mikhailovskii A B et al 1997 Plasma Phys. Rep. 23844

[16] Nagaoka K et al 2013 Nucl. Fusion 53072004

[17] Yamamoto S 2014 Effect of ECH/ECCD on energetic-ion driven MHD instabilities in low shear helical plasmas USJapan JIFT Meeting (Kyoto University, June 3-5)

[18] Cappa A et al 2015 Impact of ECRH on the NBI-driven Alfvén activity in the TJ-II stellarator: experiments and data analysis 14th CWGM (Warsaw)

[19] Valovic M et al 2000 Nucl. Fusion 401569

[20] Ding X T et al 2013 Nucl. Fusion 53043015

[21] Evans J D et al 1992 Phys. Rev. Lett. 691528

[22] Schlatter C et al 2009 33rd EPS Conf. on Plasma Physics vol 30I (Rome, 19-23 June 2006) (ECA) p 1.149

[23] Schneider P A et al 2015 Rev. Sci. Instrum. 86073508

[24] Eriksson L-G et al 1993 Nucl. Fusion 331037

[25] Hedin J et al 2002 Nucl. Fusion 42527

[26] Borba D and Kerner W 1999 J. Comput. Phys. 153

[27] Pinches A D et al 1998 Comput. Phys. Commun. 111133

[28] Lauber P et al 2007 J. Comput. Phys. 226447 